\title{
G protein-coupled estrogen receptors: novel therapeutic targets in aldosterone- induced hypertension?
}

\author{
Christopher G Sobey, Qhuyn Nhu Dinh, Antony Vinh, Grant R Drummond \\ Department of Physiology, Anatomy \& Microbiology, La Trobe University, Australia
}

Introduction. Some effects of aldosterone may be modulated by the G protein-coupled estrogen receptor 1 (GPER). Furthermore, the GPER agonist, G-1, can exert T cell-mediated anti-inflammatory actions, acutely lower blood pressure (BP), and reduce post-stroke infarct injury.

Methods. Here we tested in mice the effects of G-1 (0.03 mg/kg/d s.c.) and G-15 (GPER antagonist; $0.3 \mathrm{mg} / \mathrm{kg} / \mathrm{d}$ s.c.) on BP (using tail-cuff plethysmography) over $14 \mathrm{~d}$ in two models of hypertension: 1$)$ aldosterone $/ \mathrm{salt}(0.72 \mathrm{mg} / \mathrm{kg} / \mathrm{d} \mathrm{s.c.} \mathrm{+}$ $0.9 \% \mathrm{NaCl}$ for drinking) and 2) angiotensin II $(0.7 \mathrm{mg} / \mathrm{kg} / \mathrm{d}$ s.c. $)$; and also assessed sex differences, and the role of lymphocytes and endogenous estrogen (in ovariectomised females) in those effects. All groups were $\mathrm{n}=8$ or more.

Results. In male C57B16 mice, the aldosterone/salt-induced increase in BP $(\sim 25 \mathrm{mmHg})$ was attenuated by $\sim 50 \%$ with co-administration of G-1 $(\mathrm{P}<0.05)$. G-15 did not alter aldosterone/salt-induced hypertension in male C57B16 but prevented the anti-hypertensive effect of G-1. Moreover, whereas aldosterone/salt alone had no effect on BP in female C57B16 mice for $>7 \mathrm{~d}$, co-administration of G-15 with aldosterone/salt resulted in a prompt increase of $\sim 20 \mathrm{mmHg}$ by $\mathrm{d}$ $7(\mathrm{P}<0.05)$. In contrast, ovariectomised females resembled males in their BP profile in response to aldosterone/salt. There was virtually no effect of aldosterone/salt on BP in either male or female RAG1-deficient (RAG KO) mice, but this could be rescued by adoptive transfer of $\mathrm{T}$ cells from C57Bl6 into RAG KO $(\mathrm{P}<0.05)$. Neither G-1 nor G-15 had any effect on angiotensin II-induced hypertension in male C57Bl6 mice. T cells, as well as B cells, macrophages and neutrophils in spleen and kidneys were found to have high expression of GPER.

Conclusion. The findings suggest that aldosterone/salt-induced hypertension is strictly $\mathrm{T}$ lymphocyte-dependent and is markedly suppressed by GPER activation on these cells by endogenous estrogen or by administration of G-1. 